Document downloaded from:

http://hdl.handle.net/10251/40664

This paper must be cited as:

Parra Vega, V.; Gonzalez Garcia, B.; Seguí Simarro, JM. (2013). Morphological markers to correlate bud and anther development with microsporogenesis and microgametogenesis in pepper (Capsicum annuum L.). Acta Physiologiae Plantarum. 35(2):627-633. doi:10.1007/s11738-012-1104-x.

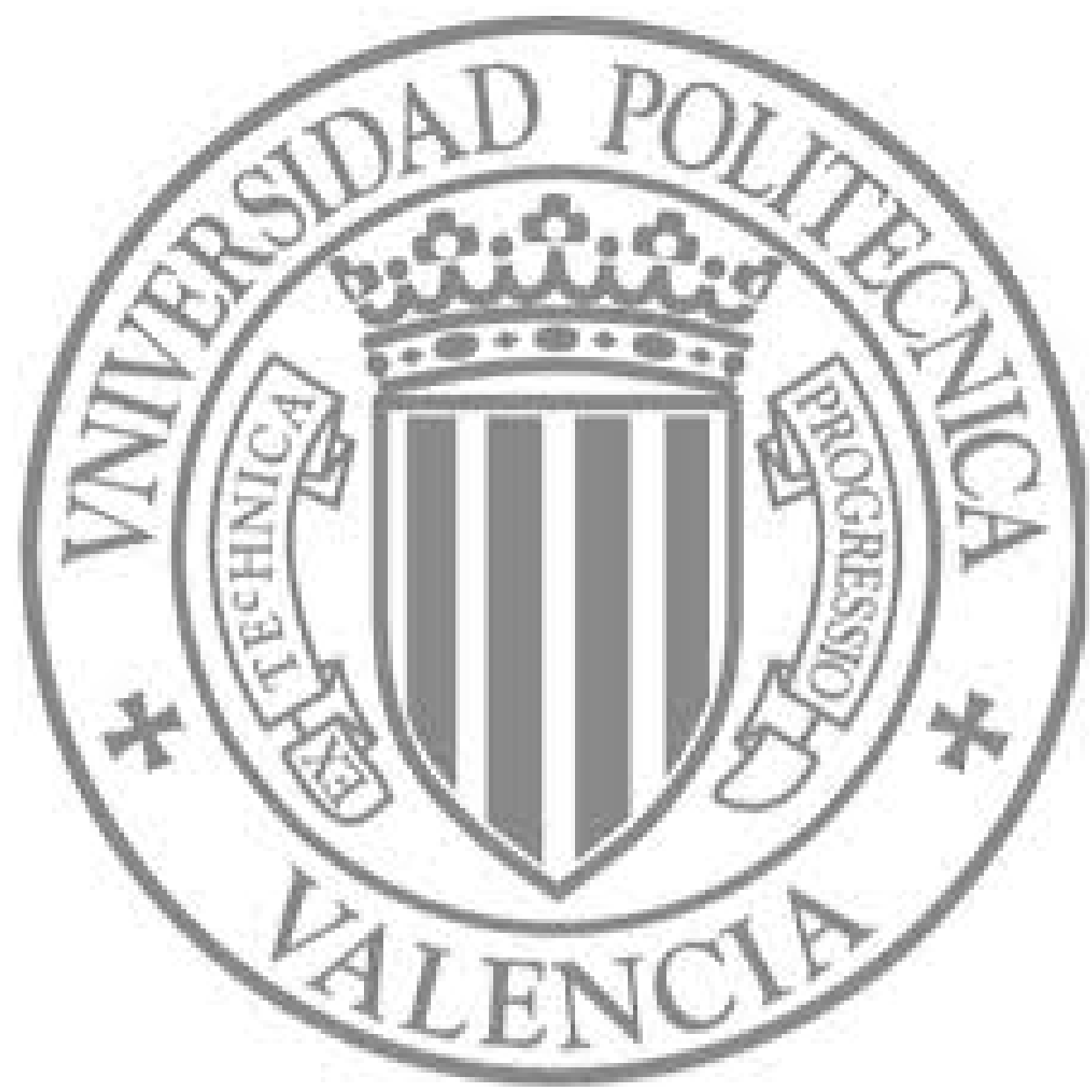

The final publication is available at

http://dx.doi.org/10.1007/s11738-012-1104-x

Copyright Springer Verlag (Germany) 


\title{
Morphological markers to correlate bud and anther development with microsporogenesis and microgametogenesis in pepper (Capsicum annuum L.)
}

\author{
Verónica Parra-Vega, Beatriz González-García and José M. Seguí-Simarro*
}

COMAV - Universitat Politècnica de València. CPI, Edificio 8E - Escalera I, Camino de Vera, s/n, 46022. Valencia, Spain.

* Corresponding author

Tel./Fax: +34 963879047

e-mail: seguisim@btc.upv.es.

Keywords: anther length, bud length, flower development, microsporogenesis, microgametogenesis, morphological markers.

\begin{abstract}
The identification of microspores or pollen grains at particular developmental stages during micrporogenesis or microgamentogenesis is an important step for different basic and applied purposes. Among them, the most relevant example from a biotechnological perspective is the production of androgenic doubled haploids. For this and other techniques, precise, fast, easy and reliable criteria to identify flower buds carrying microspores or pollen at particular stages are essential. In anthocyaninproducing pepper types, the particularities of flower development allow for the identification of several morphological markers potentially useful as criteria for such an identification. In this work, our aim was to determine the easiest and more accurate criterion to correlate visible, measurable traits of bud and anther development with each of the individual stages of microsporogenesis and microgametogenesis. For this, we used three Spanish sweet pepper F1 hybrids ('Herminio', 'Gacela' and 'Águila'). We analyzed and discussed the accuracy and practical usefulness of using anther length, bud length, anther purple pigmentation and the ratio between calyx length and bud length (calyx/bud ratio) as predictors of individual microspore/pollen developmental stages. According to our results, we propose a combination of calyx/bud ratio and anther
\end{abstract}


pigmentation as an easy, fast and accurate criterion potentially applicable to anthocyanin-producing pepper cultivars to determine their particular markers.

\section{Introduction}

During microsporogenesis and microgametogenesis, development of the microspore and the pollen grain is paralleled with changes in the anther and in the flower bud. These changes relate to bud and anther size, shape and color, among others. The study of these correlative changes are important for the identification of visible morphological markers that allow us to infer what are the developmental stages of the microspores/pollen grains contained within an anther, in a non invasive manner. In other words, with no need for excision and opening of the corresponding flower bud. These morphological markers may be useful for different practical applications where microspores/pollen at particular developmental stages must be identified and used. For example, for developmental studies of microsporogenesis and microgametogenesis where samples at different stages must be isolated to extract proteins or RNA, or to be observed with a microscope. Other examples with a more applied perspective could include techniques that need the isolation of pollen for storage or for viability or vigour tests, which include the isolation and in vitro culture of mature pollen grains to promote the development of pollen tubes (Shivanna 2003). However, among the biotechnological applications of these morphological markers, the most important would be the identification of buds and anthers with microspores/pollen at the optimal stage to be induced towards androgenesis.

Induction of androgenesis is the most convenient way to obtain doubled haploids, which are valuable tools in breeding programs. For an efficient production, either through anther culture or through isolated microspore culture, it is essential to have a protocol previously optimized. In such a protocol, a central issue is the accurate selection of donor flower buds containing microspores or pollen grains at the right stage of development. For the vast majority of inducible species, it is widely agreed that induction can only be achieved in a narrow time frame revolving around the first pollen mitosis (Seguí-Simarro 2010). This means that buds and anthers containing mostly late, vacuolate microspores (VM) and just divided, young bicellular pollen (YBP) must be 
precisely identified. This is not a trivial issue, since out of this time frame induction efficiency drops dramatically or is nearly impossible (Seguí-Simarro 2010). Thus, it is essential to identify suitable morphological markers, predictors of the precise developmental stage of the microspore or pollen grain within the anther. However, this is not always easy, since different species have different flower anatomies, with different sizes, shapes and colors for their different organs. This also applies to different cultivars within a species.

Pepper (Capsicum annuum) has been traditionally considered a species recalcitrant to androgenesis induction. Anther cultures have been used in a number of cultivars with relative efficiency (reviewed in Irikova et al. 2011; Seguí-Simarro et al. 2011), but successful application of microspore cultures has only been reported in few hot (asian) pepper types (Kim et al. 2008; Supena et al. 2006b). Nevertheless, the stages most sensitive to inductive treatments in pepper revolve around the VM and YBP (Irikova et al. 2011), exactly the same described for most of the known inducible species. Pepper is not an exception in this sense. In pepper, the search for suitable morphological markers has been approached from different perspectives. The length of specific flower whorls has been used by some authors to estimate bud size. The most frequently measured whorls have been corolla (Dunwell 2010) and calyx, where the ratio between calyx and corolla has been the most used parameter (Koleva-Gudeva et al. 2007; Ltifi and Wenzel 1994; Mityko et al. 1995; Nowaczyk and Kisiala 2006). Other authors opted for a direct estimation of the degree of purple antocyanin pigments that anthers of many pepper types accumulate during their development (Regnet 1994; Kim et al. 2008; Kim et al. 2004) or by a combination of this marker with the calyx/corolla ratio (Barany et al. 2005; Barany et al. 2010; Buyukalaca et al. 2004; Dumas de Vaulx et al. 1981; Supena et al. 2006b; Supena et al. 2006a; Ercan et al. 2006; Lantos et al. 2009). From these reports it can be concluded that there is no a wide consensus with respect to the most suitable marker to define which pepper flower buds should be used to maximize the percentage of VM and YBP. In this work we addressed this issue in sweet pepper. Our goal was to determine the easiest and more accurate morphological markers to correlate the visible changes of anthers and buds during flower development with the corresponding stages of the microspores and pollen grains contained in them. To approach this, we first characterized the changes undergone by buds and anthers during microspore and pollen development in three Spanish sweet pepper cultivars 
('Herminio', 'Gacela' and 'Águila'). According to the described changes, we evaluated the correlation of different morphological criteria (anther length, bud length, pigmentation level of anthers and calyx/bud ratio) with microspore/pollen development, and the convenience of using them to identify anthers enriched in a particular developmental window: the transitional stage of the first pollen mitosis (from VM to YBP). We chose these stages due to the importance of their precise identification for anther and microspore culture experiments.

\section{Material and methods}

\section{Plant material}

The following three anthocyanin-producing, commercial F1 hybrids of pepper (Capsicum annuum L.) were used: 'Herminio' (Lamuyo type), 'Gacela' and 'Águila' (California type), all of them from Syngenta Seeds. Plants were grown in a growth chamber at $25^{\circ} \mathrm{C}$ under controlled conditions of humidity (60\%) and photoperiod (16/8) during 12 consecutive months from October to September.

\section{Microspore stage determination}

Flower buds ranging from 2 to $8 \mathrm{~mm}$ long were selected and excised manually. Bud, anther (two anthers per bud) and calyx lengths were measured with an electronic digital caliper. For buds, measurements were performed from the pedicel insertion point to the tip of the bud, as illustrated in Figure 1A. For anthers, measurements were performed from the filament insertion (basal end) to the anther tip (apical end), as illustrated in Figure 1B. Anther purple pigmentation was categorized into four pigmentation levels as illustrated in Figure 1C: (1) no pigmentation, (2) pigments in the apical end, (3) pigments covering most of the distal surface and only traces at the borders of the proximal surface of the anthers, and (4) full pigmentation of the distal surface and almost full pigmentation of the proximal surface of the anthers. For calyx/bud ratio, calyx length, considered from pedicel insertion to the topmost part of the calyx ( Figure 1D) was divided by the corresponding bud length.

A minimum of ten buds were considered for each of the intervals used. For each bud, the parameters above described were measured. From them, two anthers were measured, their pigmentation level estimated, and then used for microspore/pollen 
staging. When different, the length of both anthers was averaged. The different microspore/pollen developmental stages present in a single anther were observed and counted with a Zeiss (Axiovert 40 CFL) inverted microscope. A minimum of 300 randomly chosen microspores/pollen grains per anther preparation were counted. For each anther, the total microspores/pollen population was categorized into seven stages, as illustrated in Figure 2, according to the staging published for other solanaceous species (Seguí-Simarro and Nuez 2005; Salas et al. 2012): (1) meiocytes and tetrads, (2) young microspores, (3) mid microspores, (4) vacuolate microspores, (5) young, (6) mid and (7) mature bicellular pollen grains. For each stage, percentages from the total population were calculated. For simplification, the young and mid microspore stages were joined into a single stage $(\mathrm{YM}+\mathrm{MM})$. Numerical data were processed and graphically represented using a Microsoft Excel spreadsheet.

\section{Results and discussion}

First, we studied the parallel development of microspores/pollen grains (Figures 2A-G), flower buds (Figures 2A'-G') and anthers (Figures 2A'’-G'), covering from the meiocyte to the mature pollen stages. Dividing meiocytes or tetrads, characterized by four independent microspores still enclosed within the post-meiotic cell walls (Figure 2A), were observed in all flower buds, which were rounded and fully covered by sepals (Figure 2A'). At this stage, corresponding anthers were small and green (Figure 2A'). Young microspores, just released from the tetrad, showed a spherical shape and a still thin pollen coat (Figure 2B). At this stage, buds remained rounded and closed (Figure 2B'). Anthers slightly elongated with respect to the previous stage, but with a similar shape and color (Figure 2B',). Mid microspores (Figure 2C) showed marked differences with young microspores, mainly consisting on the lobulation of the thicker, polygonal and more sculptured coat, which began to be evident. These changes correlated with changes in the corresponding buds (Figure 2C'), which increased in size. Besides, sepals began to open in the still rounded bud, allowing for the observation of the corolla at the top of the bud. Anthers (Figure 2C')' increased in size as well, while keeping similar morphologies and colors. Vacuolate (mature) microspores presented a thicker exine wall where apertures were clearly visible (Figure 2D). At this stage, flower buds continued their increase in size and petals were partially visible, with sepals covering approximately three-fourths of the bud (Figure 2D'). Anthers turned to dark yellow and 
presented a slightly purple pigmentation at the apical end, only on their distal, petal facing side (Figure 2D')). The transition of VMs to YBP (Figure 2E) was characterized by changes in bud size, a widening of the bud receptacle, and the clear emergence of petals out of the calyx (Figure 2E'). Purple pigmentation of the distal side of the yellow anthers was rather evident at this stage (Figure 2E'). At the mid bicellular pollen stage (Figure 2F), enlarged buds began to elongate due to petal growth, which reached a size approximately equal to that of sepals (Figure 2F'). Anthers became purple through most of their distal surface (Figure 2F')' Finally, at the mature pollen stage, these enlarged, densely filled grains (Figure 2G) correlated with long buds, where the exposed petal length usually exceeded that of sepals (Figure 2G'), and anthers were fully purple at the distal side and the borders of the proximal side (Figure 2G'). Further developmental stages consisted on anthesis, petal growth and anther dehiscence, with no changes in pollen grains other than desiccation (data not shown).

Next, we assessed the convenience of using anther length as a criterion. We correlated the evolution of microsporogenesis and microgametogenesis with anther increases in length in the 'Herminio' (Figure 3A), 'Gacela' (Figure 3B) and 'Águila' (Figure 3C) cultivars. The percentage of microspores/pollen at each developmental stage is represented by the size of the corresponding bubble. These figures evidenced that anther grows in parallel to microspore development, and also to pollen development up to the mid pollen stage, where anthers did not grow further. Shorter (younger) anthers were characterized by the coexistence of microspores at few different stages, with one of them clearly predominating. In contrast, heterogeneity was more characteristic of longer (older) anthers, with up to five stages in 3.00-3.50 mm anthers of cvs. 'Herminio' and 'Gacela'. Cues of small bubbles, corresponding to low percentages of microspores, could be observed in older anthers of the three genotypes. They were most likely indicating the presence of dead and/or delayed microspores, unable to develop synchronously with the rest of microspores. With respect to the identification of the first pollen mitosis interval, we looked for the ranges containing mostly VM and YBP (green bubbles in Figure 3). In case of doubt, we selected the range with a majority of VM with respect to $\mathrm{YBP}$, since they were at a stage immediately prior to mitosis, and would enter it soon. 'Herminio' anthers contained these stages at a length range from 2.50 to $2.99 \mathrm{~mm}$ (Figure 3A), whereas in 'Gacela' and 'Águila' it was from 2.0 to $2.49 \mathrm{~mm}$ (Figures 3B, 3C). Such a slight discrepancy 
(0.5 mm) could be attributable to genotype-dependent differences in growth, and it is likely that by using less restrictive length intervals, differences would disappear. In light of these results, it was clear that anther length correlates with microspore/pollen development with very slight variations between cultivars, confirming that anther length can be used to accurately identify microspore/pollen developmental stages, at least up to the mid pollen stage.

Then, we focused on the usefulness of bud length as a convenient criterion. From a practical perspective, this parameter would be faster and easier to measure. However, bud length might not correlate with anther growth and microspore/pollen development. For example, this occurs in related species such as tomato or eggplant, where sepals grow considerably at the onset of bud development, while anthers do not grow at the same pace (Seguí-Simarro and Nuez 2005; Salas et al. 2012). Thus, a previous mandatory study consisted on the verification of the relationship between anther and bud lengths. We studied this by analyzing the linear correlation between anther and bud length values for all three cultivars. In all cases, a positive linear correlation was clearly observed, with linear regression coefficients (R) higher than 0.9 (data not shown). Then, we compared bud lengths with the different stages of microsporogenesis and microgametogenesis (Figures 3D-F). Due to the larger size of buds with respect to anthers, larger $(1 \mathrm{~mm})$ length intervals were established. In this case, a clear correlation was also observed at the different stages, confirming bud length as another reliable criterion. With respect to the VM and YBP stages, the three cultivars ('Herminio' in Figure 3D, 'Gacela' in Figure 3E and 'Águila' Figure 3F) presented the highest percentages at the interval ranging from to 4.0 to $4.99 \mathrm{~mm}$. Thus, a length between 4 and 5 mm could be an accurate marker to identify 'Herminio', 'Gacela' and ‘Águila’ buds with highest percentages of VM and YBP.

The correlation between anther purple pigmentation patterns and microspore/pollen development was subsequently studied in a way similar to that described above for anther and bud lengths. As observed in Figure 3G for 'Herminio', Figure 3H for 'Gacela' and Figure 3I for 'Águila', pigmentation patterns also correlated well with microspore/pollen development. According to the pigmentation levels established in Materials and methods, the higher percentage of VM and YBP was observed exclusively in anthers with a level 2 of pigmentation (purple pigmentation at 
the apical end of the distal surface) for all the genotypes studied. Thus, the differential anther pigmentation pattern would also be a valid criterion to identify anthers enriched in VM and YBP.

As the last morphological criterion, we assessed the convenience of using the proportion of calyx length with respect to the whole bud. This criterion allows for a quick visual identification of suitable buds, provided that their exact values are studied for each stage. As for bud length, it was necessary to verify whether this parameter correlated with microspore/pollen development. For this, we analyzed the linear correlation between bud length and calyx/bud ratio values for all three cultivars. In all cases, a clearly positive linear correlation was observed, with linear regression coefficients (R) ranging between 0.84 and 0.92 (Figures 3J-L). Therefore, the calyx/bud ratio could also be a reliable criterion to be used. Since the highest concentration of VM and YBP was found at the bud length interval of 4.0 to $4.99 \mathrm{~mm}$, we extrapolated the corresponding calyx/bud ratios for the three cultivars and obtained a similar calyx/bud ratio between 0.8 and 0.9 (red arrows in Figure 3J for 'Herminio', Figure 3K for 'Gacela' and Figure 3L for 'Águila'). Thus, buds with a calyx covering about 80-90\% of the total bud length should be selected to maximize the presence of VM and YBP.

If we consider that externally visible criteria to identify microspore/pollen stages must be precise, all four criteria analyzed would be valid, being anther and bud length the most precise. However, criteria should also be easy to use and quickly measurable. According to this, bud and anther length would not be the most useful in practice due to the time needed to excise and measure every single bud and anther. These timeconsuming procedures would delay significantly the experiments aimed to do with the microspores/pollen, which may in turn compromise their viability. The small size of pepper anthers would be an added difficulty. Furthermore, these invasive processes imply that after measurement, many useless excised and open buds will be discarded, thus reducing the efficiency of these procedures. Anther pigmentation has been suggested as the most reliable criterion for anthocyanin-producing cultivars, since it is less genotype-dependent (Regner 1996). However, it is based on the visual estimation of pigmentation, which may reduce its accuracy, and is a time-consuming and destructive procedure as well. All this considered, these three markers would have little practical utility. For a large-scale practical implementation of a criterion to identify 
microspores/pollen, it is preferable to rely on easier and faster criteria, even if they imply less accuracy. Besides, it would be desirable to apply this criterion on the buds still in planta, avoiding excision. According to this, the calyx/bud ratio would be the best criterion. Compared to others, its only limitation would be a reduced accuracy, since in practice it may be difficult to visually distinguish between ratios of $70 \%, 80 \%$ or $90 \%$. Although for an experienced observer this may not be a problem, we propose in case of doubt a combination of the two external criteria hereby described, calyx/bud ratio and anther pigmentation, as follows. First, optimal buds could be quickly identified in planta according to their calyx/bud ratio. Then, once in the laminar flowhood, anthers could be excised and quickly checked on the basis of their pigmentation pattern. Restricting selected anthers to those matching both criteria would compensate for the reduced accuracy of these criteria when used separately.

In conclusion, we propose that the combined use of calyx/bud ratio and anther pigmentation may provide easy, fast and accurate identification of buds and anthers with microspores/pollen at particular developmental stages in anthocyanin-producing pepper cultivars. In the cultivars used in this study and for the identification of VM and YBP stages, this would mean the selection of anthers from buds having a calyx/bud ratio of about $80-90 \%$, and then the exclusion of those not having purple pigments only at the apical end of the distal surface.

\section{Author contribution}

Verónica Parra-Vega and Beatriz González García performed all the experimental work required for this study. Jose M. Seguí-Simarro was responsible for the experimental design, interpretation of results and writing of the manuscript.

\section{Acknowledgements}

We acknowledge Dr. Rosa Peiró, Mrs. Nuria Palacios and Mrs. Patricia Corral for their valuable help, as well as the staff of the COMAV greenhouses. VPV is a predoctoral fellow of the FPU program of the Spanish Ministry of Education. This work was supported by grant from Spanish Ministry of Science and Innovation (MICINN) AGL2010-17895 to JMSS. 


\section{References}

Barany I, Fadon B, Risueno MC, Testillano PS (2010) Cell wall components and pectin esterification levels as markers of proliferation and differentiation events during pollen development and pollen embryogenesis in Capsicum annuum L. J Exp Bot 61:1159-1175. doi:10.1093/jxb/erp392

Barany I, Gonzalez-Melendi P, Fadón B, Mityko J, Risueño MC, Testillano PS (2005) Microspore-derived embryogenesis in pepper (Capsicum annuum L.): subcellular rearrangements through development. Biol Cell 97:709-722

Buyukalaca S, Comlekcioglu N, Abak K, Ekbic E, Kilic N (2004) Effects of silver nitrate and donor plant growing conditions on production of pepper (Capsicum annuum L.) haploid embryos via anther culture. European Journal of Horticultural Science 69 (5):206-209

Dumas de Vaulx R, Chambonnet D, Pochard E (1981) Culture in vitro d'anthères de piment (Capsicum annuum L.): amèlioration des taux d'obtenction de plantes chez différents génotypes par des traitments à $+35^{\circ} \mathrm{C}$. Agronomie 1 (10):859-864

Dunwell JM (2010) Haploids in flowering plants: origins and exploitation. Plant Biotechnol J 8 (4):377-424. doi:10.1111/j.1467-7652.2009.00498.x

Ercan N, Sensoy FA, Sirri Sensoy A (2006) Influence of growing season and donor plant age on anther culture response of some pepper cultivars (Capsicum annuum L.). Sci Hort 110 (1):16-20

Irikova T, Grozeva S, Rodeva V (2011) Anther culture in pepper (Capsicum annuum L.) in vitro. Acta Physiol Plant 33 (5):1559-1570. doi:10.1007/s11738-0110736-6

Kim M, Jang I-C, Kim J-A, Park E-J, Yoon M, Lee Y (2008) Embryogenesis and plant regeneration of hot pepper ( Capsicum annuum L.) through isolated microspore culture. Plant Cell Rep 27 (3):425-434

Kim M, Kim J, Yoon M, Choi D-I, Lee K-M (2004) Origin of multicellular pollen and pollen embryos in cultured anthers of pepper (Capsicum annuum). Plant Cell Tissue and Organ Culture 77:63-72

Koleva-Gudeva LR, Spasenoski M, Trajkova F (2007) Somatic embryogenesis in pepper anther culture: The effect of incubation treatments and different media. Sci Hort 111 (2):114-119

Lantos C, Juhász A, Somogyi G, Ötvös K, Vági P, Mihály R, Kristóf Z, Somogyi N, Pauk J (2009) Improvement of isolated microspore culture of pepper (Capsicum annuum L.) via co-culture with ovary tissues of pepper or wheat. Plant Cell Tiss Org Cult 97 (3):285-293. doi:10.1007/s11240-009-9527-9

Ltifi A, Wenzel G (1994) Anther culture of hot and sweet pepper (Capsicum annuum L.): influence of genotype and plant growth temperature. Capsicum Eggplant Newsl 13:74-77

Mityko J, Andrasfalvy A, Csillery G, Fari M (1995) Anther culture response in different genotypes and F1 hybrids of pepper (Capsicum Annuum L). Plant Breed 114 (1):78-80

Nowaczyk P, Kisiala A (2006) Effect of selected factors on the effectiveness of Capsicum annuum L. anther culture. J Appl Genetics 47 (2):113-117

Regner F (1996) Anther and microspore culture in Capsicum. In: Jain SM, Sopory SK, Veilleux RE (eds) In vitro haploid production in higher plants, vol 3. Kluwer Academic, Dordrecht, The Netherlands, pp 77-89 
Regnet F (1994) Microspore culture of Capsicum annuum. Capsicum Eggplant Newsl 13 (1114):69-70

Salas P, Rivas-Sendra A, Prohens J, Seguí-Simarro JM (2012) Influence of the stage for anther excision and heterostyly in embryogenesis induction from eggplant anther cultures. Euphytica 184 (2):235-250. doi:10.1007/s10681-011-0569-9

Seguí-Simarro JM (2010) Androgenesis revisited. Bot Rev 76 (3):377-404. doi:10.1007/s12229-010-9056-6

Seguí-Simarro JM, Corral-Martínez P, Parra-Vega V, González-García B (2011) Androgenesis in recalcitrant solanaceous crops. Plant Cell Rep 30 (5):765-778. doi:10.1007/s00299-010-0984-8

Seguí-Simarro JM, Nuez F (2005) Meiotic metaphase I to telophase II is the most responsive stage of microspore development for induction of androgenesis in tomato (Solanum lycopersicum). Acta Physiol Plant 27 (4B):675-685

Shivanna KR (2003) Pollen biology and biotechnology. Science Publishers Inc., Enfield, NH, USA

Supena EDJ, Muswita W, Suharsono S, Custers JBM (2006a) Evaluation of crucial factors for implementing shed-microspore culture of Indonesian hot pepper (Capsicum annuum L.) cultivars. Sci Hort 107 (3):226-232

Supena EDJ, Suharsono S, Jacobsen E, Custers JBM (2006b) Successful development of a shed-microspore culture protocol for doubled haploid production in Indonesian hot pepper (Capsicum annuum L.). Plant Cell Rep 25 (1):1-10

\section{Figure legends}

Figure 1: Criteria used to measure the four morphological parameters used in this study. A: Bud length. B: Anther length. C: Anther pigmentation. D: Calyx length. Anthers in B and C are presented at their distal, petal-facing side (D) and proximal, pistil-facing side $(\mathrm{P})$. See text for further details.

Figure 2: Changes during microspore/pollen (A-G), bud (A'-G'), and anther development (A”-G’). A: meiocytes and tetrads. B: Young microspores. C: Mid microspores. D: Vacuolate microspores. E: Young bicellular pollen. F: Mid pollen. G: Mature pollen. Anthers are presented in A' -G', at their distal (left) and proximal sided (right). Bars: A-G: $10 \mu \mathrm{m}$; A'-G’’: $1 \mathrm{~mm}$.

Figure 3: Correlation of anther length (A-C), bud length (D-F), anther pigmentation (G-I) and calyx/bud ratio (J-L) with microspore/pollen development in cvs 'Herminio’ (A, D, G, J), ‘Gacela’ (B, E, H, K) and ‘Águila’ (C, F, I, L). Percentages of microspores/pollen at each stage are proportionally represented by bubbles. Green bubbles correspond to the stages where most vacuolate microspores and young bicellular pollen are found. For figures $\mathrm{J}$-L, blue dots are the data points used to 
calculate the regression line. $\mathrm{M}+\mathrm{T}$ : meiocytes and tetrads. $\mathrm{YM}+\mathrm{MM}$ : Young and mid microspores. VM: Vacuolate microspores. YBP: Young bicellular pollen. MP: Mid pollen. MAP: Mature pollen. 

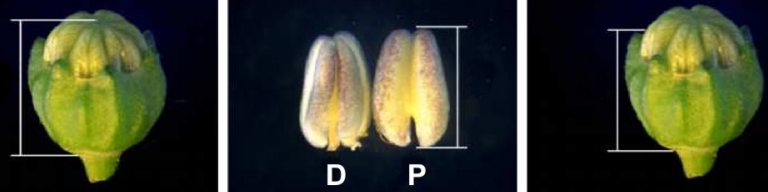

C

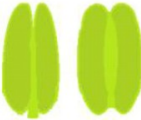

Level 1

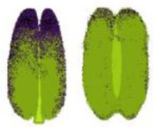

Level 2

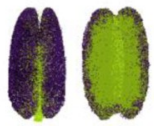

Level 3

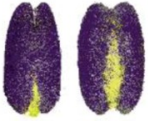

Level 4 


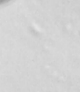

(ㄷ)
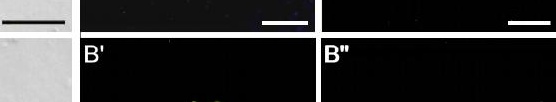

C

E

(2)

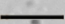

D

(3)

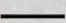

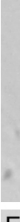

-

(2)
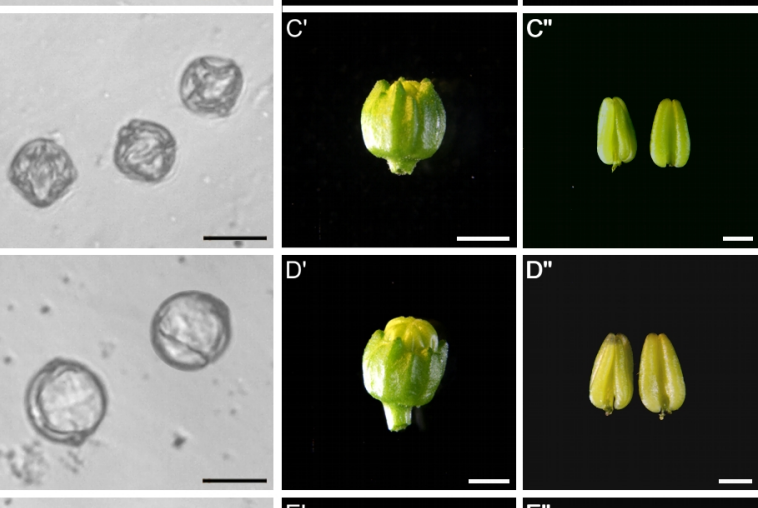

F

$\mathrm{F}$
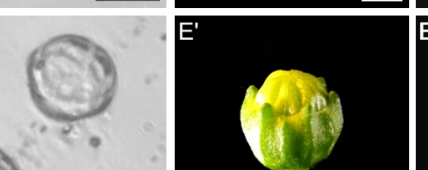

E'

ad

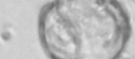

o e the
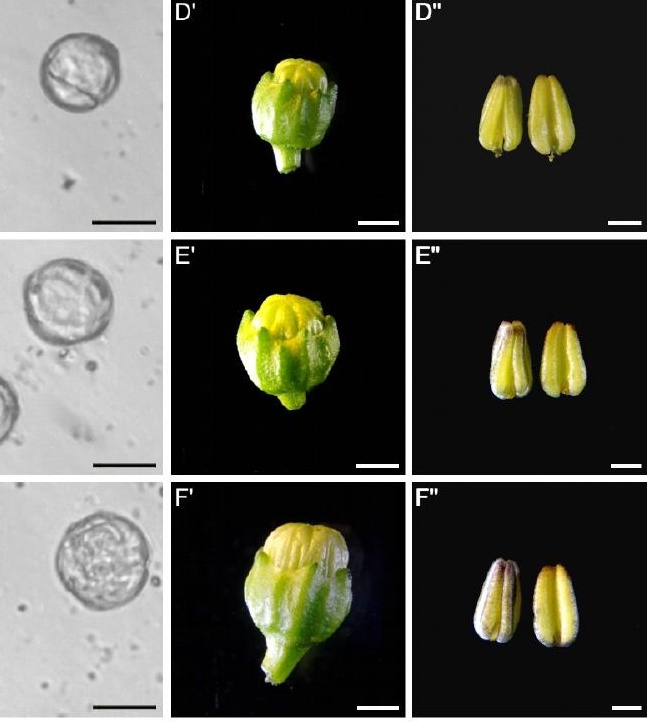

G
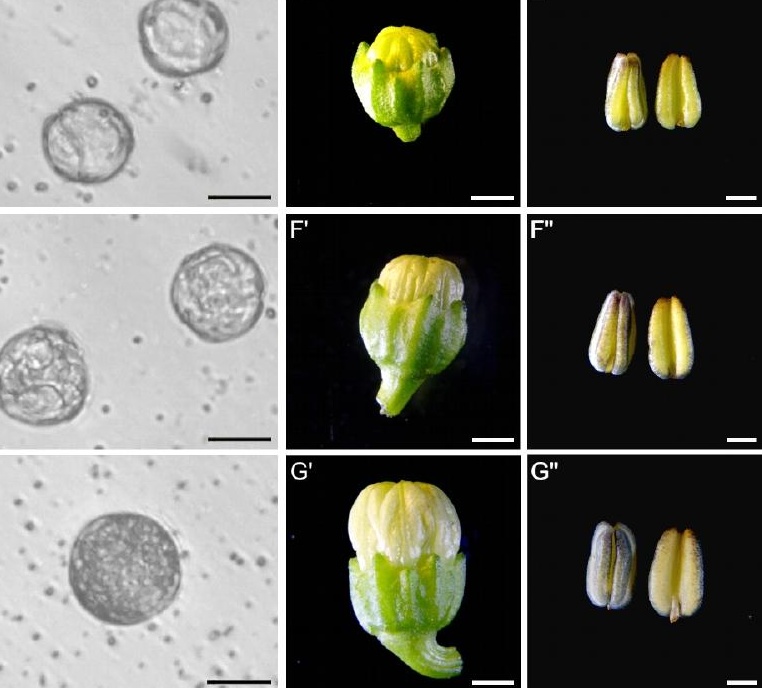

$F^{\prime \prime}$

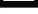


